\title{
ON THE LOCATION OF THE ZEROS OF ANALYTIC FUNCTIONS
}

\author{
K.K. DEWAN \\ Department of Mathematics \\ Jamia Millia University \\ New Delhi - 110025 \\ India \\ N.K. GOVIL \\ Department of Algebra, Combinatorics and Analysis \\ Division of Mathematics \\ Auburn University \\ Auburn, AL 36849 \\ U.S.A. \\ (Recelved May 1, i989)
}

\begin{abstract}
In this paper we obtain reglons containing all the zeros of a class of analytic functions whose coefficients are subject to certain conditions. Our results sharpen some of the results known in this direction. Also we give some examples to show that in some cases the regions obtalned by our results are considerably sharper than the reglons obtained by known results.
\end{abstract}

KEY WORDS AND PHRASES. Zeros of polynomials, rational functions, and other analytic functions.

1980 AMS SUBJECT CLASSIFICATION CODE. $30 \mathrm{C15}$.

1. INTRODUCTION AND STATEMENT OF RESULTS.

In this paper we obtain the regions containing all the zeros of certain classes of analytic functions. Our results improve some of the results known in this direction. We shall in fact be proving the following results which sharpen some of the results of Az1z and Mohammad [1] and of Govil and Rahman [2]. 
THEUREM 1. Let $f(z)=\sum_{j=0}^{\infty} a_{j} z^{j} \neq 0$, be analytic in $|z| \leq t$.

If $\left|a r g a_{j}\right| \leq \alpha \leq \pi / 2, j=0,1,2, \ldots$, and for some finite nonnegative integer $k,\left|a_{0}\right| \leq t\left|a_{1}\right| \leq \cdots \leq t^{k}\left|a_{k}\right| \geq t^{k+1}\left|a_{k+1}\right| \geq \cdots$, then $f(z)$ does not van1sh in $|z|<R_{1}$,

where

$$
R_{1}=\frac{-t^{2}|b|\left(M_{1}-\left|a_{0}\right| t\right)+\left\{t^{4}|b|^{2}\left(M_{1}-\left|a_{0}\right| t\right)^{2}+4 t^{3}\left|a_{0}\right| M_{1}{ }^{3}\right\}^{\frac{1}{2}}}{2 M_{1}^{2}} .
$$

Here

$$
M_{1}=t\left|a_{0}\right|\left\{\left(\frac{2\left|a_{k}\right|}{\left|a_{0}\right|} t^{k}-1\right) \cos \alpha+\sin \alpha+\frac{2 \sin \alpha}{\left|a_{0}\right|} \sum_{j=1}^{\infty} t^{j}\left|a_{j}\right|\right\}
$$

and

$$
b=a_{0}-t a_{1}
$$

The above theoren sharpens Theorem 6 of Aziz and Mohammad [1]. If we take $k=0$ in Theorem 1, we get the following corollary which sharpens Corollary 5 of AzIz and Mohammad [1].

COROLLARY 1. Let $f(z)=\int_{j=0}^{\infty} a_{j} z^{j}$ be analytic in $|z| \leq t$. If $\left|\arg a_{j}\right| \leq \alpha \leq \pi / 2, j=0,1,2, \ldots$, and $\left|a_{o}\right| \geq t\left|a_{1}\right| \geq t^{2}\left|a_{2}\right| \geq \ldots$, then $f(z)$ does not vanish in

$$
|z|<R_{2} \text {, }
$$

where

$$
R_{2}=\frac{-t^{2}|b|\left(M_{2}-t\left|a_{0}\right|\right)+\left\{t^{4}|b|^{2}\left(M_{2}-t\left|a_{0}\right|\right)^{2}+t^{3}\left|a_{0}\right| M_{1}{ }^{3}\right\}^{\frac{1}{2}}}{2 M_{2}{ }^{2}} .
$$

Here

and

$$
M_{2}=t\left|a_{0}\right|\left(\cos \alpha+\sin \alpha+\frac{2 \sin \alpha}{\left|a_{0}\right|} \sum_{j=1}^{\infty} t^{j}\left|a_{j}\right|\right)
$$

$$
b=a_{0}-t a_{1}
$$

For $t=1$, the above corollary sharpens Theorem 3 of Govil and Rahman [2].

THEOREM 2. Let $f(z)=\sum_{j=0}^{\infty} a_{j} z^{j}$ be analytic in $|z| \leq t$. If Re $a_{j}=\alpha_{j}$, and Im $a_{j}=\beta_{j}, j=0,1,2, \ldots$, and for some finite nonnegative integer $k$, $\alpha<\alpha_{0} \leq t \alpha_{1} \leq \cdots \leq t^{k} \alpha_{k} \geq t^{k+1} \alpha_{k+1} \geq \cdots$, then $f(z)$ does not vanish in

$$
|z|<R_{\urcorner},
$$


where

$$
R_{3}=\frac{-t^{2}|b|\left(M_{3}-\left|a_{0}\right| t\right)+\left(t^{4}|b|^{2}\left(M_{3}-\left|a_{0}\right| t\right)^{2}+4 t^{3}\left|a_{0}\right|_{3}^{3}\right\}^{\frac{1}{2}}}{2 M_{4}^{2}}
$$

Here

$$
M_{3}=t \alpha_{0}\left\{2\left(\frac{\alpha_{\alpha}}{\alpha_{0}}\right) t^{k}-1+\frac{2}{\alpha_{0}} \sum_{j=0}^{\infty} t^{j}\left|\beta_{j}\right|\right\}
$$

and

$$
b=a_{0}-t a_{1}
$$

This theorem sharpens Theorem 7 of $\mathrm{Aziz}$ and Mohammad [1]. If we take $k=0$ in the above Theorem 2, we get the following corollary which generalizes and sharpens Theorem 4 of Govil and Rahman [2].

COROLlaRY 2. Let $f(z)=\sum_{j=0}^{\infty} a_{j} z^{j}$ be analytic in $|z| \leq t . \quad$ If $a_{j}=\alpha_{j}+i \beta_{j}, j=0,1,2, \ldots$ and $0<\alpha_{0} \geq t \alpha_{1} \geq t^{2} \alpha_{2} \geq \ldots$, then $f(z)$ does not vanish in

$$
|z|<R_{4}
$$

where

$$
R_{4}=\frac{-t^{2}|b|\left(M_{4}-\left|a_{0}\right| t\right)+\left\{t^{4}|b|^{2}\left(M_{4}-t\left|a_{0}\right|^{2}+4 t^{3}\left|a_{0}\right|_{M^{3}}\right\}^{\frac{1}{2}}\right.}{2 M_{4}^{2}} .
$$

Here

$$
M_{4}=\operatorname{ta}_{0}\left(1+\frac{2}{\alpha_{0}} \sum_{j=0}^{\infty}\left|\beta_{j}\right| t^{j}\right) \text {, }
$$

and

$$
b_{0}=a_{0}-t a_{1}
$$

As remarked earlier, Theorems 1 and 2 are respectively the refinements of Theorems 6 and 7 of AzIz and Mohammad [1]. For the sake of completeness we will verify that Theorem 1 sharpens Theorem 6 of Aziz and Mohammad [1], for which it is sufficient to show that

$$
\begin{aligned}
\frac{t}{M} \leq R_{1} & =\frac{-t^{2}|b|\left(M_{1}-\left|a_{0}\right| t\right)+\left\{t^{4}|b|^{2}\left(M_{1}-\left|a_{0}\right| t\right)^{2}+4 M_{1}{ }^{3} t^{3}\left|a_{0}\right|^{\frac{1}{2}}\right.}{2 M_{1}{ }^{2}} \\
& =\frac{\left.-t^{3}|b||a|(M-1)+t^{3}\left|a_{0}\right| t|b|^{2}(M-1)^{2}+4\left|a_{0}\right|^{2} M^{3}\right\}^{\frac{1}{2}}}{2 t^{2}\left|a_{0}\right|^{2} M^{2}},
\end{aligned}
$$

because as can be easily seen, $M_{1}=t\left|a_{0}\right| M$. Now (1.14) is equivalent to

$$
2\left|a_{0}\right| M<-|b|(M-1)+\left\{|b|^{2}(M-1)^{2}+4\left|a_{0}\right|^{2} M^{3}\right\}^{\frac{1}{2}},
$$

which is true if and only if 


$$
(M-1)\left(\left|a_{0}\right| M-|b|\right) \geq 0,
$$

and since (1.15) is evidently true by Cauchy's inequality [3, p. 84], the inequality (1.13) is verified.

The fact that Theorem 2 is a refinement of Theorem 7 of [1] can be verified on the same lines and we therefore onit the proof.

In general Theorems 1 and 2 give results which are at least as good as obtainable from Theorems 6 and 7 of Az1z and Mohammad [1], but in some cases the results obtalned by Theorems 1 and 2 are significantly better than those obtained respectively from Theorem 6 and 7 of Az1z and Mohanmad [1]. We 1llustrate this by the following examples.

EXAMPLE 1.

$f(z)=1+\left(\frac{1}{22}+\frac{1}{22}\right) z+\left(\frac{1}{82}+\frac{1}{82}\right) z^{2}+\left(\frac{1}{322}+\frac{1}{32}\right) z^{3}+\ldots$

If we take $t=2, k=1, \alpha=\pi / 4$, then Theorem 6 of Az1z and Mohammad [1] gives that $f(z)$ does not vanish in $|z|<.4714045$, while our Theorem 1 gives that $f(z)$ does not vanish in $|z|<1 \cdot 1185882$.

EXAMPLE 2.

$$
\begin{aligned}
f(z)=(1+1) & +\left(2+\frac{1}{2}\right) z+\left(3+\frac{1}{2^{2}}\right) z^{2}+\left(4+\frac{1}{2^{3}}\right) z^{3}+\left(1+\frac{1}{2^{4}}\right) z^{4}+\left(1+\frac{1}{2^{5}}\right) z^{5} \\
& +\left(1+\frac{1}{2^{6}}\right) z^{6}+\cdots
\end{aligned}
$$

If we take $t=1, k=3$ then Theorem 7 of Aziz and Mohammad [1] gives that $f(z)$ does not vanish in $|z|<0.333 \ldots$ while by our Theorem 2, $f(z)$ does not vanish in $|z|<0.791$.

2. LEMMAS.

We need the following lemmas.

LEMMA 1. If $f(z)$ is analytic in $|z| \leq 1, f(0)=a$ where $|a|<1, f^{\prime}(0)=b$, $|f(z)| \leq 1$ on $|z|=1$, then for $|z| \leq 1$,

$$
|f(z)| \leq \frac{(1-|a|)|z|^{2}+|b||z|+|a|(1-|a|)}{|a|(1-a \mid)|z|^{2}+|b||z|+(1-|a|)}
$$

The example $f(z)=\frac{a+\frac{b}{1+a} z-z^{2}}{1-\frac{b}{1+a} z-a z^{2}}$ shows that the estimate is sharp.

Above lemma is due to Govil et al [4].

One gets easily from the above lemma, the following.

LEMMA 2. If $f(z)$ is analytic in $|z| \leq R, f(0)=0, f^{\prime}(0)=b$ and $|f(z)| \leq M$ for $|z|=R$, then for $|z| \leq R$,

$$
|f(z)| \leq \frac{M|z|}{R^{2}} \frac{M|z|+R^{2}|b|}{M+|z||b|} \text {. }
$$

LEMMA 3. Let $f(z)=\sum_{j=0}^{\infty} a_{j} z^{j} \equiv 0$ be analytic in $|z| \leq t$.

If $\left|\arg a_{j}\right| \leq \alpha \leq \pi / 2, j=0,1,2, \ldots$, then 


$$
\left|t a_{j}-a_{j-1}\right| \leq|t| a_{j}|-| a_{j-1}|| \cos \alpha+\left(t\left|a_{j}\right|+\left|a_{j-1}\right|\right) \sin \alpha \text {. }
$$

The proof is omitted as it follows ininediately from the inequality (6) in [2].

3. PROOFS UF THE THEUREMS.

Proof of Theorem 1. Clearly $\lim _{j \rightarrow \infty} t^{j} a_{j}=0$. We consider the function

$$
\begin{aligned}
& F(z)=(z-t) f(z) \\
& =-t a_{0}+z \sum_{j=1}^{\infty}\left(a_{j-1}-t a_{j}\right) z^{j-1} \\
& =-t a_{0}+G(z) \text {, say. }
\end{aligned}
$$

Using Lemma 3, we get for $|z|=t$,

$$
\begin{aligned}
|G(z)| & \leq t\left(\sum_{j=1}^{\infty}|t| a_{j}|-| a_{j-1}|| t^{j-1} \cos \alpha+\sum_{j=1}^{\infty}\left(t\left|a_{j}\right|+\left|a_{j-1}\right|\right) t^{j-1} \sin \alpha\right) \\
& =t\left(\cos \alpha \sum_{1}^{k}\left(t\left|a_{j}\right|-\left|a_{j-1}\right|\right) t^{j-1}+\sum_{k+1}^{\infty}\left(\left|a_{j-1}\right|-t\left|a_{j}\right|\right) t^{j-1} \cos \alpha\right. \\
& \left.+2 \sin \alpha \sum_{j=1}^{\infty} t^{j}\left|a_{j}\right|+\left|a_{0}\right| \sin \alpha\right) \\
& =t\left|a_{0}\right|\left\{\left(\frac{2\left|a_{k}\right| t^{k}}{\left|a_{o}\right|}-1\right) \cos \alpha+\sin \alpha+\frac{2 \sin \alpha}{\left|a_{0}\right|} \sum_{j=1}^{\infty} t^{j}\left|a_{j}\right|\right\} \\
& =t\left|a_{0}\right| M, \text { say } \\
& =M_{1} .
\end{aligned}
$$

Since $G(0)=0, G^{\prime}(0)=a_{0}-t a_{1}=b$, we get by Lemma 2 , that for $|z| \leq t$,

$$
|G(z)|<\frac{M_{1}|z|}{t^{2}} \cdot \frac{M_{1}|z|+t^{2}|b|}{M_{1}+|b||z|}
$$

Combining (3.1) and (3.2), we get for $|z| \leq t$,

$$
\begin{aligned}
|F(z)| \geq t\left|a_{0}\right|-|G(z)| & \\
& \geq t\left|a_{0}\right|-\frac{M_{1}|z|}{t^{2}} \cdot \frac{M_{1}|z|+t^{2}|b|}{M_{1}+|b||z|} \\
& =\frac{-1}{t^{2}\left(M_{1}+|b||z|\right)}\left\{|z|^{2} M_{1}{ }^{2}+t^{2}|b||z|\left(M_{1}-t\left|a_{0}\right|\right)-t^{3}\left|a_{0}\right|^{M_{1}}\right\} \\
& >0,
\end{aligned}
$$


if

$$
\begin{aligned}
& |z|<\frac{-t^{2}|b|\left(M_{1}-t\left|a_{0}\right|\right)+\left[t^{4}|b|^{2}\left(M_{1}-t\left|a_{0}\right|\right)^{2}+4 t^{3}\left|a_{0}\right| M_{1}{ }^{3}\right\}^{\frac{1}{2}}}{2 M_{1}{ }^{2}} \\
& \quad=R_{1} .
\end{aligned}
$$

It is easy to verify that $R_{1} \leq t$. Thus in $|z| \leq t,|F(z)|>0$ if $|z|<R_{1}$. Consequently $f(z)$ does not vanish in $|z|<R_{1}$ and the proof of Theorem 1 is complete.

Proof of Theorem 2. Clearly $\underset{j \rightarrow \infty}{\lim } \alpha_{j} t^{j}=0, \lim _{j \rightarrow \infty} P_{j} t^{j}=0$. As before we consider the function $F(z)=(z-t) f(z)=-t_{0}+G(z)$, where $G(z)$ is same as in (3.1). Then on $|z|=t$, we have

$$
\begin{aligned}
|G(z)| & \leq t \sum_{j=1}^{\infty}\left|a_{j-1}-t a_{j}\right| t^{j-1} \\
& \leq t\left(\sum_{j=1}^{\infty}\left|\alpha_{j-1}-t{ }_{j}\right| t^{j-1}+\sum_{j=1}^{\infty}\left(\left|\beta_{j-1}\right|+t\left|\beta_{j}\right|\right)\right. \\
& =t \alpha_{0}\left\{2\left(\sum_{\alpha_{0}}^{\alpha_{k}}\right) t^{k}-1+\left(\frac{2}{\alpha_{0}}\right) \sum_{j=0}^{\infty} t^{j}\left|\beta_{j}\right|\right\} \\
& =M_{3} .
\end{aligned}
$$

Since $G(0)=0, G^{\prime}(0)=a_{0}-t a_{1}=b$, we can apply Lemma 2 to the function $G(z)$ and then proceeding on the lines of the proof of Theorem 1, the proof of Theorem 2 can be completed.

REFERENCES

1. Aziz, A. and Mohammad, Q. G., On the zeros of a certain class of polynomlals and related analytic functions, J. Math. Anal. App1. 75(1980), 495-502.

2. Govil, N. K. and Rahman, Q. I., On the Eneström-Kakeya theorem, Tôhoku Math. J. 20(1968), 126-136.

3. Titchmarsh, E. C., The Theory of Functions, 2nd edition, Oxford University Press, 1939.

4. Govil, N. K., Rahman, Q. I., and Schmeisser, G., On the derivative of a polynomia 1, Illino1s J. Math. 23(1979), 319-329. 


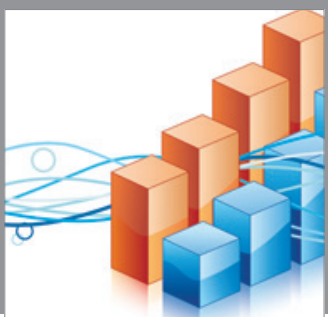

Advances in

Operations Research

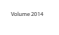

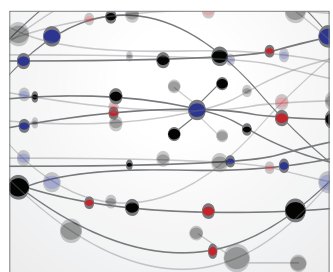

\section{The Scientific} World Journal
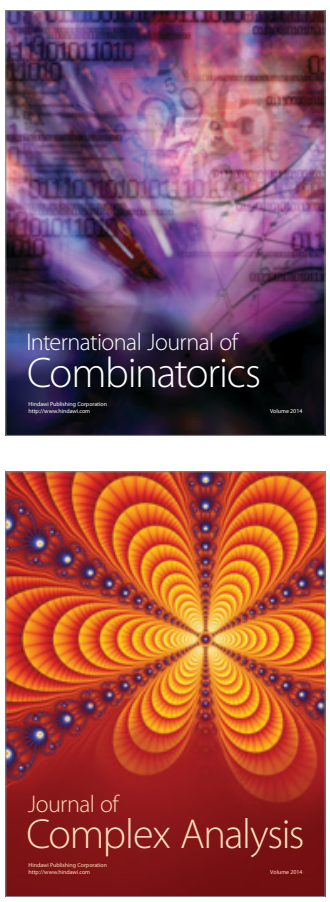

International Journal of

Mathematics and

Mathematical

Sciences
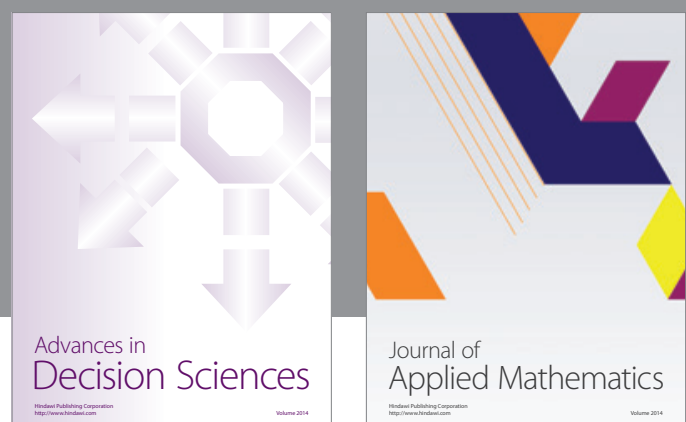

Journal of

Applied Mathematics
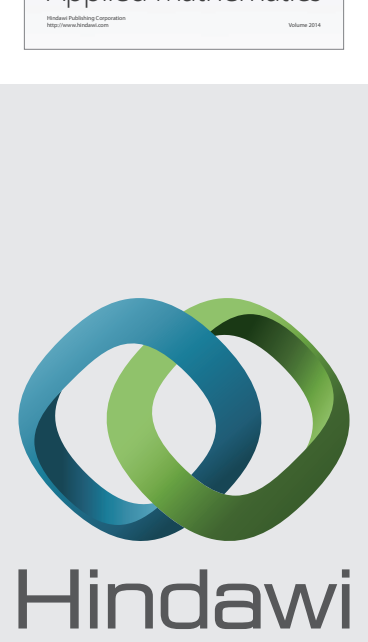

Submit your manuscripts at http://www.hindawi.com
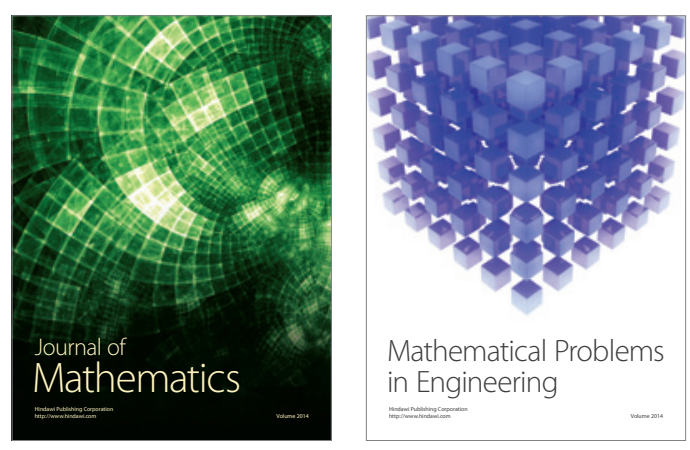

Mathematical Problems in Engineering
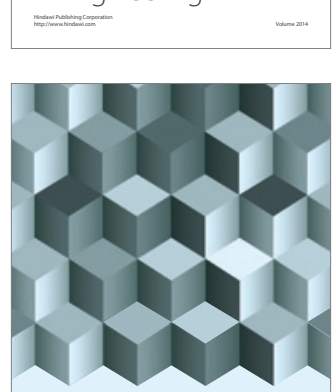

Journal of

Function Spaces
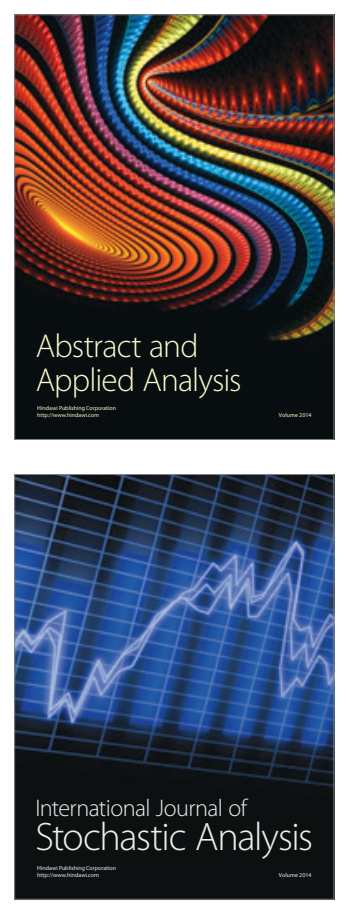

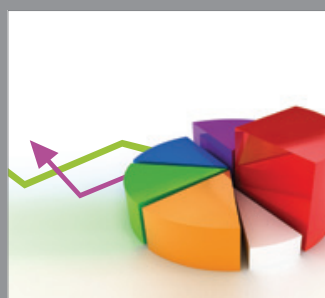

ournal of

Probability and Statistics

Promensencen
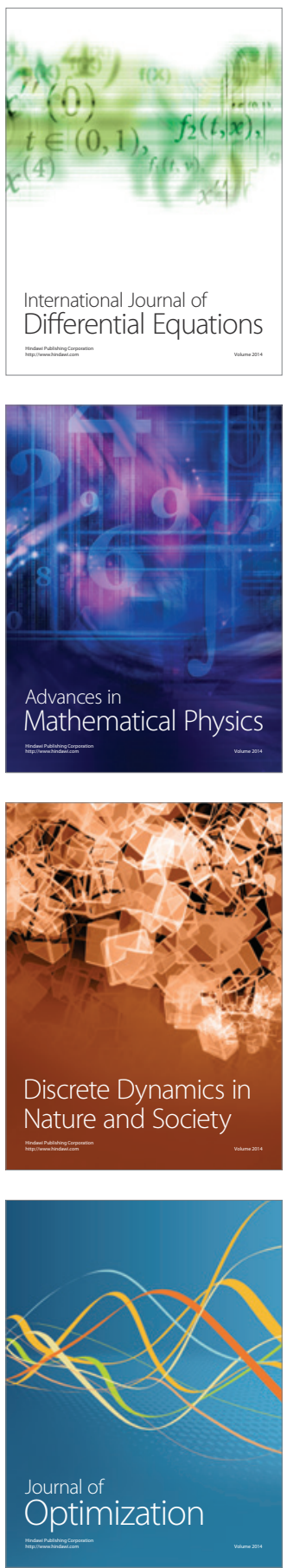